\title{
Tests of Various Scintillator Detectors in Selected Mono-Energetic Neutron Beams
}

\author{
Aleš Jančár̆ $\check{r}^{1}$, Jiří Čulen ${ }^{1}$, Filip Mravec ${ }^{1}$, Michal Koštál ${ }^{3}$, Daniel Dlhopolček ${ }^{2}$, Elisa Pirovano ${ }^{4}$, Ralf \\ Nolte $^{4}$, František Cvachovec ${ }^{2}$, Václav Přenosil ${ }^{2}$, Zdeněk Matěj ${ }^{2}$ \\ ${ }^{1}$ VF, a.s., Svitavská 588, 67921 Černá Hora, Czech Republic \\ ${ }^{2}$ Masaryk University, Botanická 68a, 61200 Brno, Czech Republic \\ ${ }^{3}$ Research Center Řež Ltd, 25068 Husinec-Řež 130, Czech Republic \\ ${ }^{4}$ Physikalisch-Technische Bundesanstalt, Bundesallee 100, 38116 Braunschweig, Germany \\ ales.jancar@vfnuclear.com
}

\begin{abstract}
In this paper we study quality of three types of organic scintillators - stilbene, p-terphenyl and EJ-299-33. We used monoenergetic neutron fields with a wide range of neutron energies in PTB Braunschweig. All the tests were carried out with NGA-01 spectrometer. The results of the measurements are evaluated spectra from the spectrometer.

We discuss the quality of each scintillator, such as the FWHM of the peaks.

Keywords -Scintillation detectors, Mono-energetic neutron sources, Pulse-shape discrimination, FWHM, Digital-signal processing.
\end{abstract}

\section{INTRODUCTION}

$\mathrm{T}$ HIS paper presents preliminary results from measurements at the PTB facility in Germany. Several monoenergetic neutron beams were used together with three scintillators connected to fast digitizer NGA-01: stilbene, p-terphenyl and EJ-299-33 [5].

\section{EXPERIMENTAL SETUP}

The irradiations were performed at the PTB Ion Accelerator Facility, where monoenergetic neutron fields are produced via selected reactions of proton and deuteron beams with light or medium-weight target nuclei. The measurements were carried out in open geometry in the low-scattering hall where the contribution of room-return neutrons is minimized by having grid floors $[1,2]$. The three neutron energies considered in this campaign 1.5, 2.5 and $19 \mathrm{MeV}$, see Tab. 1 .

\section{TABLE I}

MEAN NEUTRON ENERGY $\mathrm{E}_{\mathrm{N}}$ OBTAINED AT THE NEUTRON EMISSION ANGLE OF ZERO DEGREES RELATIVE TO THE DIRECTION OF THE INCIDENT BEAM, FOR THE GIVEN TARGETS AND ION BEAM (PROJECTILE) ENERGIES $\mathrm{E}_{\mathrm{PROJ}}$.

\begin{tabular}{llll}
\hline \hline $\mathrm{E}_{\text {proj }}[\mathrm{keV}]$ & Target & Reaction & En $[\mathrm{MeV}]$ \\
\hline 2336 & $\mathrm{Ti}(\mathrm{T}), 961 \mu \mathrm{g} / \mathrm{cm}^{2}$ & ${ }^{3} \mathrm{H}(\mathrm{p}, \mathrm{n})^{3} \mathrm{He}$ & 1.5 \\
3350 & $\mathrm{Ti}(\mathrm{T}), 1831 \mu \mathrm{g} / \mathrm{cm}^{2}$ & ${ }^{3} \mathrm{H}(\mathrm{p}, \mathrm{n})^{3} \mathrm{He}$ & 2.5 \\
2676 & $\mathrm{Ti}(\mathrm{T}), 1831 \mu \mathrm{g} / \mathrm{cm}^{2}$ & ${ }^{3} \mathrm{H}(\mathrm{d}, \mathrm{n})^{4} \mathrm{He}$ & 19.0 \\
\hline \hline
\end{tabular}

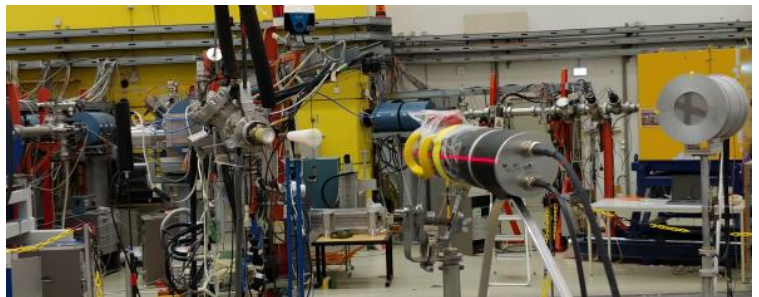

Fig. 1. Position of the detector (on the right-front) and the beam target (on the left-rear) as used for the measurements. In between there is a shadow cone.

Neutron-Gamma spectrometric system NGA-01 has been used for the measurements [4]. The input analog signal from the detector was digitized by two fast A/D converters working with a sampling frequency of $500 \mathrm{MHz}$. Digital signal processing is implemented in FPGA Xilinx Virtex-6. Integration method based on the pulse charge comparison was implemented in FPGA. Separation parameters for various scintillators calculated with this method are shown below in PSD matrices. Hamamatsu R329-02 photomultiplier was used for these measurements.

${ }^{137} \mathrm{Cs}$ and ${ }^{60} \mathrm{Co}$ sources were used for calibration.

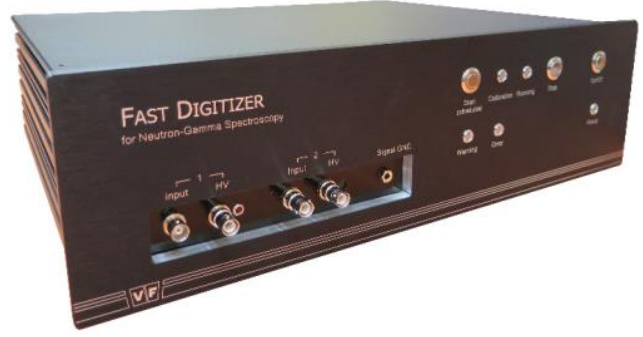

Fig. 2. Neutron gamma analyser NGA-01.

\section{EVALUATION}

NGA-01 has been used for the measurements of the apparatus proton recoil spectra. These spectra were evaluated using Maximum Likelihood Estimation method of Expectation Maximization [3]. Response functions needed for this evaluation were calculated with Monte Carlo simulation code using ENDF/B-VII.1 nuclear data.

For the evaluation of the measurements we developed spectrometric software. MCNP simulations of response 
functions using ENDF/B-VII.1 nuclear data were used. Neutron spectral flux densities are displayed.

The $19 \mathrm{MeV}$ field contained parasitic low-energy neutrons from $\mathrm{D}(\mathrm{d}, \mathrm{n}), \operatorname{Ti}(\mathrm{d}, \mathrm{n})$ and $\operatorname{Ag}(\mathrm{d}, \mathrm{n})$ reactions which were not subtracted using a non-tritiated target. For this further analysis will be performed.

\section{RESULTS}

Stilbene scintillators of the sizes of $10 \times 10 \mathrm{~mm}$ and $45 \times 45$ $\mathrm{mm}$ have been used for measurements of neutron energies of $1.5,2.5$ and $19 \mathrm{MeV}$. In all measurements corresponding peaks are identified in evaluated spectra, Fig. 3 - 14. Measurements with neutron energies of 2.5 and $19 \mathrm{MeV}$ were carried out with EJ-299-33 scintillator (Fig. 15 - 18) and with p-terphenyl scintillator (Fig. 19 - 22). We compared spectra from EJ-29933 and p-terphenyl scintillators with $45 \times 45 \mathrm{~mm}$ stilbene scintillator (shown in dotted gray line in Fig. 17, 18, 21, 22). Arbitrary units have been used for the y-axis in the graphs of evaluated spectra. Tab. 2 shows resolution values calculated from evaluated spectra.

TABLE II

THE ENERGY RESOLUTION FWHM/E FOR NEUTRON ENERGIES 2.5 AND $19 \mathrm{MEV}$.

\begin{tabular}{lllll}
\hline \hline $\mathrm{E}_{\mathrm{n}}[\mathrm{MeV}]$ & $\begin{array}{l}\text { Stilbene } 45 \\
\mathrm{~mm}\end{array}$ & EJ-299-33 & p-terphenyl & $\begin{array}{l}\text { Stilbene } 10 \\
\mathrm{~mm}\end{array}$ \\
\hline $2.5 \mathrm{MeV}$ & 0.216 & 0.361 & 0.280 & 0.232 \\
$19.0 \mathrm{MeV}$ & 0.102 & 0.184 & 0.184 & 0.063 \\
\hline \hline
\end{tabular}

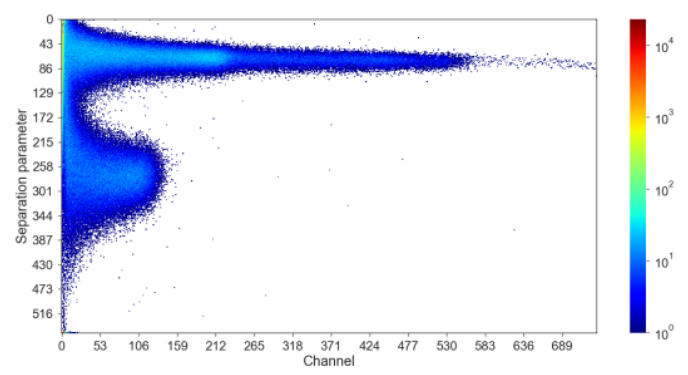

Fig. 3. PSD matrix for $1.5 \mathrm{MeV}$ measurement with stilbene $10 \times 10 \mathrm{~mm}$.

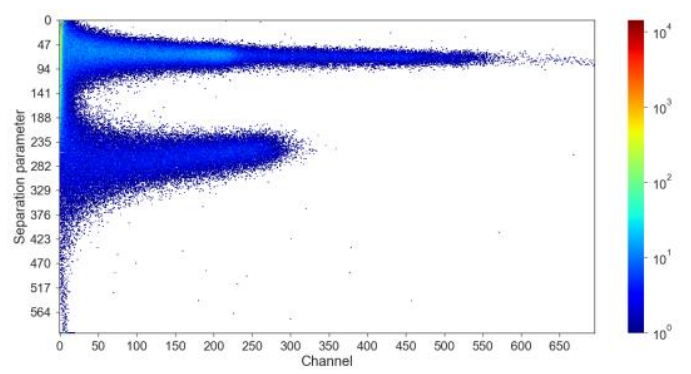

Fig. 4. PSD matrix for $2.5 \mathrm{MeV}$ measurement with stilbene $10 \times 10 \mathrm{~mm}$.

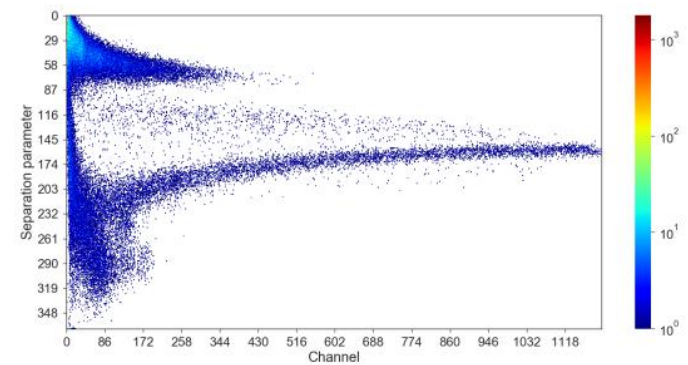

Fig. 5. PSD matrix for $19 \mathrm{MeV}$ measurement with stilbene 10x10 mm.

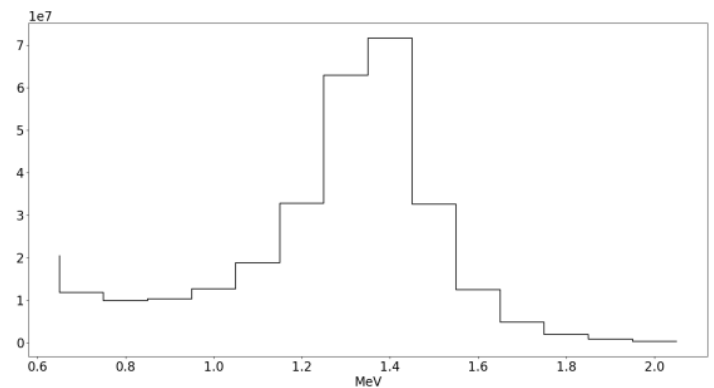

Fig. 6: Neutron spectral flux density for $10 \times 10 \mathrm{~mm}$ stilbene for $1.5 \mathrm{MeV}$ neutrons.

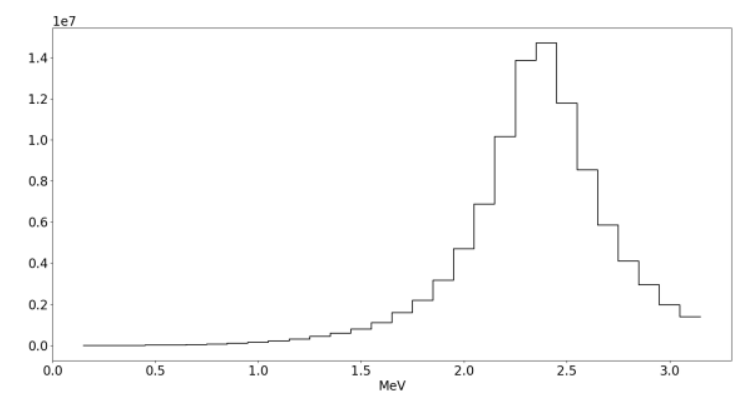

Fig. 7: Neutron spectral flux density for $10 \times 10 \mathrm{~mm}$ stilbene for $2.5 \mathrm{MeV}$ neutrons.

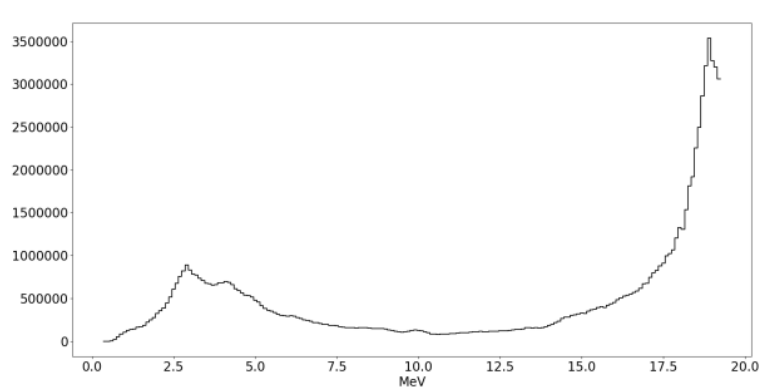

Fig. 8: Neutron spectral flux density for $10 \times 10 \mathrm{~mm}$ stilbene for $19 \mathrm{MeV}$ neutrons. 


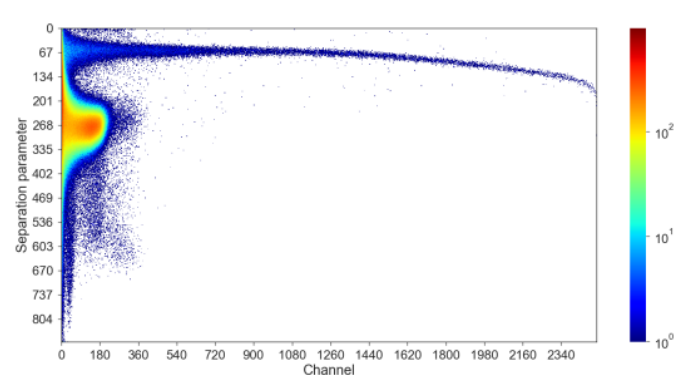

Fig. 9. PSD matrix for $1.5 \mathrm{MeV}$ measurement with stilbene $45 \times 45 \mathrm{~mm}$.

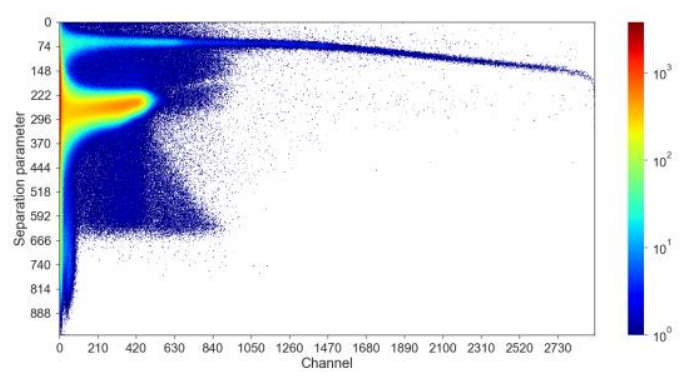

Fig. 10. PSD matrix for $2.5 \mathrm{MeV}$ measurement with stilbene $45 \times 45 \mathrm{~mm}$.

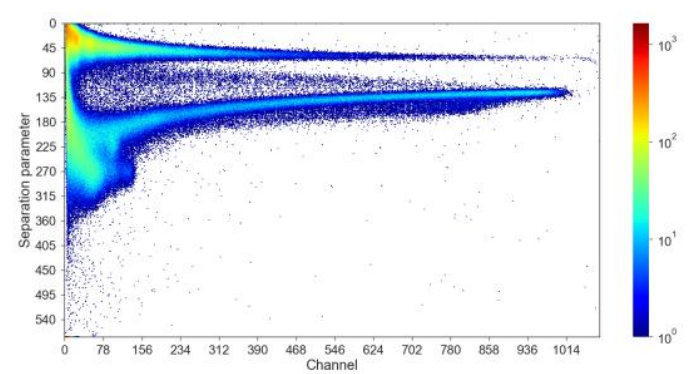

Fig. 11. PSD matrix for $19 \mathrm{MeV}$ measurement with stilbene $45 \times 45 \mathrm{~mm}$.

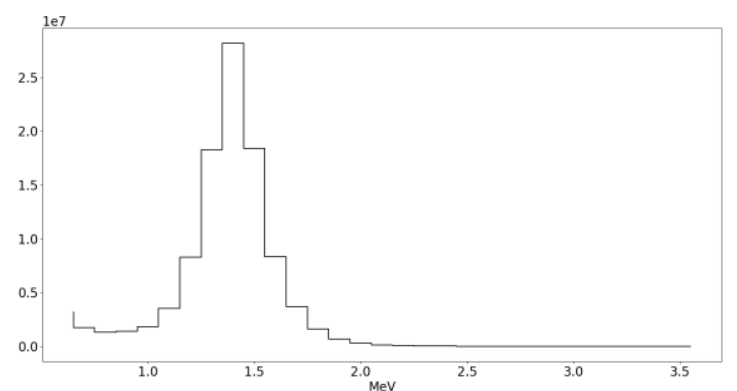

Fig. 12: Neutron spectral flux density for $45 \times 45 \mathrm{~mm}$ stilbene for $1.5 \mathrm{MeV}$ neutrons.

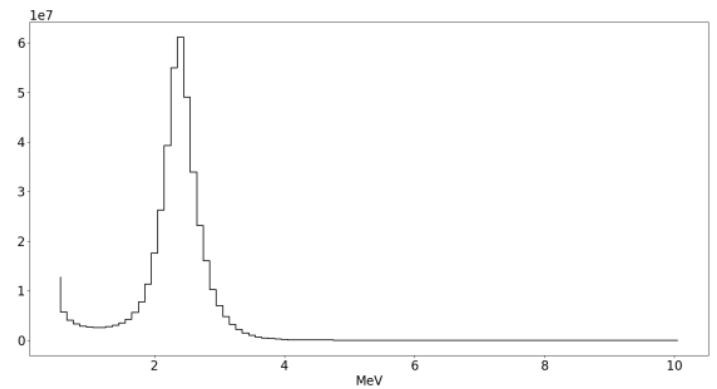

Fig. 13: Neutron spectral flux density for $45 \times 45 \mathrm{~mm}$ stilbene for $2.5 \mathrm{MeV}$ neutrons.

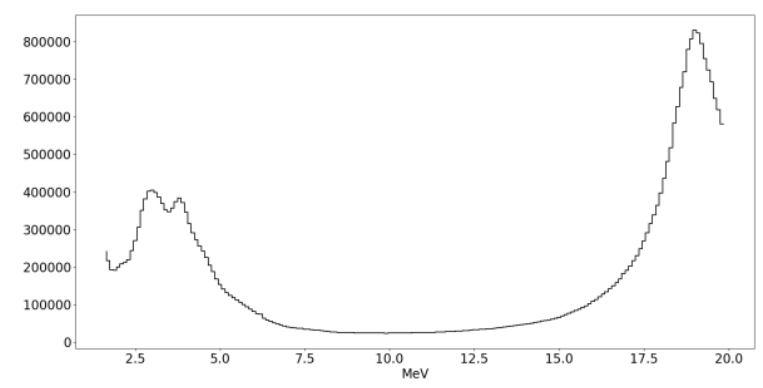

Fig. 14: Neutron spectral flux density for $45 \times 45 \mathrm{~mm}$ stilbene for $19 \mathrm{MeV}$ neutrons.

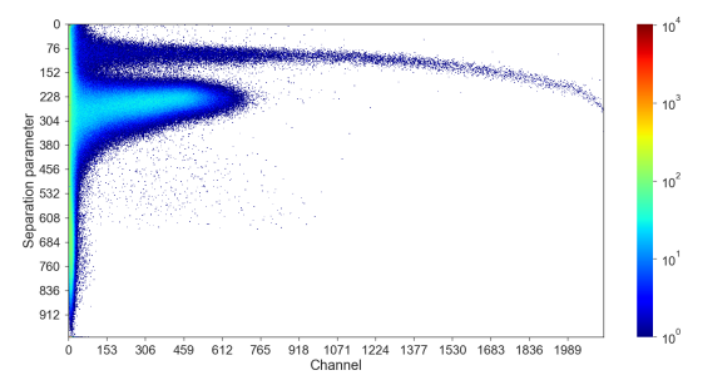

Fig. 15. PSD matrix for $2.5 \mathrm{MeV}$ measurement with 1x1 inch EJ-299-33.

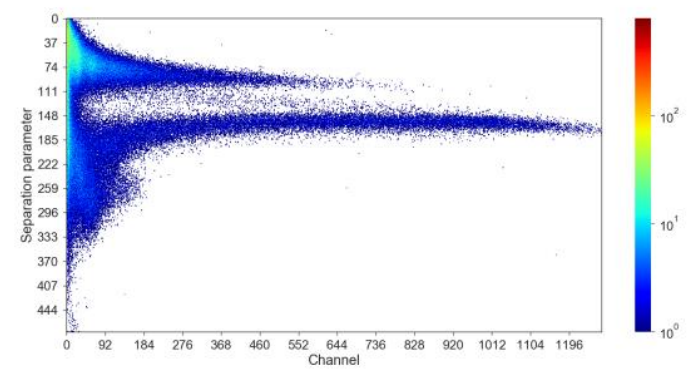

Fig. 16. PSD matrix for $19 \mathrm{MeV}$ measurement with 1x1 inch EJ-299-33. 


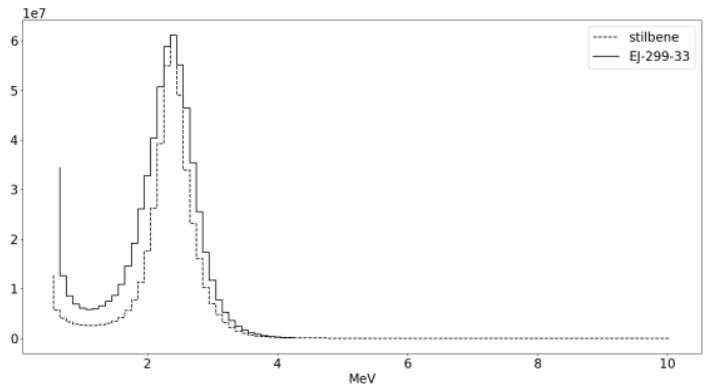

Fig. 17: Neutron spectral flux density for 1x1 inch EJ-299-33 for $2.5 \mathrm{MeV}$ neutrons. Results for stilbene $45 \times 45$ were added for comparison.

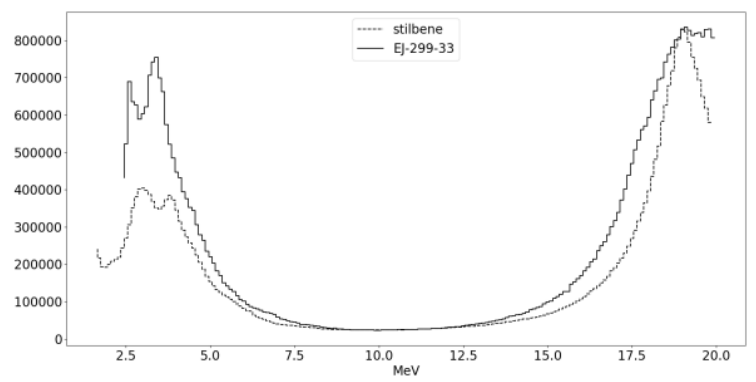

Fig. 18: Neutron spectral flux density for $1 \mathrm{x} 1$ inch EJ-299-33 for $19 \mathrm{MeV}$ neutrons. Results for stilbene $45 \times 45$ were added for comparison.

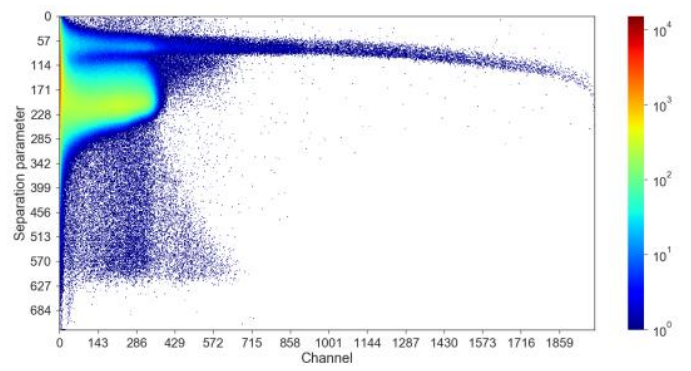

Fig. 19. PSD matrix for $2.5 \mathrm{MeV}$ measurement with p-terphenyl $45 \mathrm{x} 45 \mathrm{~mm}$

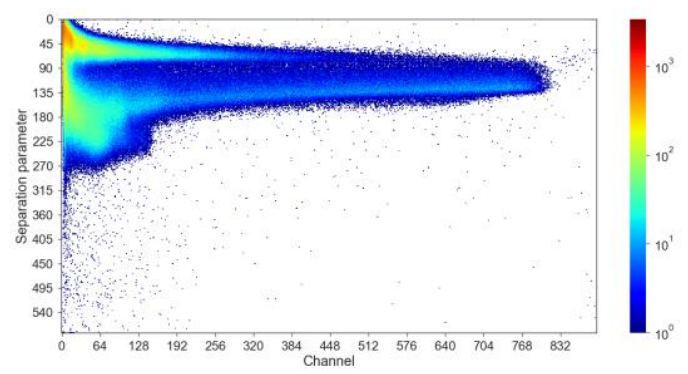

Fig. 20. PSD matrix for $19 \mathrm{MeV}$ measurement with p-terphenyl $45 x 45 \mathrm{~mm}$.

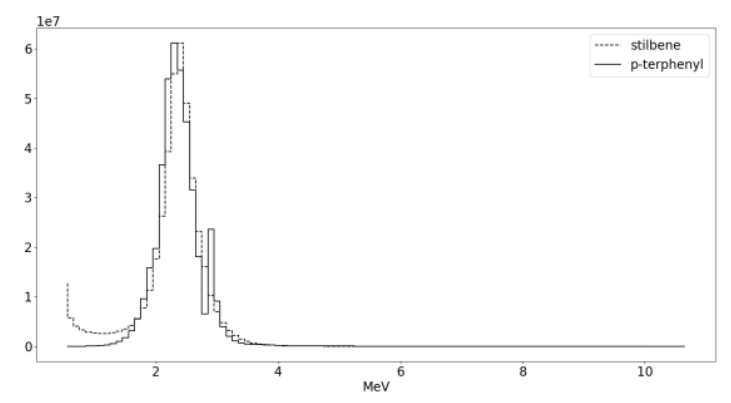

Fig. 21: Neutron spectral flux density for $45 \times 45 \mathrm{~mm}$ p-terphenyl for $2.5 \mathrm{MeV}$ neutrons. Results for stilbene $45 \times 45$ were added for comparison.

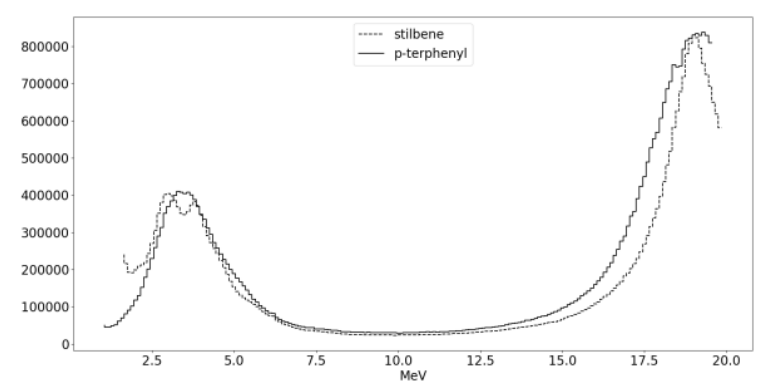

Fig. 22: Neutron spectral flux density for $45 \times 45 \mathrm{~mm}$ p-terphenyl for $19 \mathrm{MeV}$ neutrons. Results for stilbene $45 \times 45$ were added for comparison.

\section{CONCLUSIONS}

All presented scintillators show satisfactory spectrometric and pulse shape discrimination properties. Using unfolding it is possible to find characteristic peaks at expected energies.

The next step of the measurement processing would be the subtraction of the measurements with the shadow cone and with the blank target (for the $19 \mathrm{MeV}$ experiment). The data can be also used to improve the light output functions for tested scintillation materials.

\section{ACKNOWLEDGMENT}

The work presented in this paper has been supported by the research project "Methods of transmission and detection of scintillation radiation with optical fibers and energy resolution of ionizing radiation sources", No. VI20192022116 of the Ministry of the Interior of the Czech Republic and research project "Integrated spectrometric release system radioactive waste into the environment", No. FW01010063 of Technology Agency of the Czech Republic. This project has received funding from the Euratom research and training programme 2014-2018 under grant agreement No 847594 (ARIEL).

\section{REFERENCES}

[1] R. Nolte and D. J. Thomas, Monoenergetic fast neutron reference fields: I. neutron production, Metrologia, vol. 48, p. S263S273, 2011.

[2] R. Nolte and D. J. Thomas, Monoenergetic fast neutron reference fields: II. field characterization, Metrologia, vol. 48, p. S274S291, 2011.

[3] J. Cvachovec and F. Cvachovec. Maximum Likelihood Estimation of a Neutron Spectrum and Associated Uncertainties. Advances in Military Technology. Brno: Univerzita obrany, 2008, Vol. 3, No. 2, 14 s. ISSN 1802-2308.

[4] Z. Mat'ej at al., Progress in Nuclear Science and Technology, 4 (2014), p. 670 .

[5] Eljen EJ-276 scintillator [Online] Available: https://eljentechnology.com/products/plastic-scintillators/ej-276 